Requesting SAIC review of the following abstracts for presentation in one or all of the following venues:

SMRP (Society for Maintenance and Reliability Professionals) is an international organization of over 3000 members worldwide.

- HCSMRP 8th Annual Symposium Event at Moody Gardens in Galveston, TX.

- Abstracts due to planning committee by April 1, 2014.

AIAA (American Institute of Aeronautics and Astronautics)

- ATS (Annual Technical Symposium) at JSC Gilruth Houston, Texas.

- Abstracts due to planning committee by April 21, 2014

\title{
Constructing the "Best" Reliability Data for the Job
}

\section{D.L. DeMott, R.K. Kleinhammer, C.J. Kahn}

Modern business and technical decisions are based on the results of analyses. When considering assessments using "reliability data", the concern is how long a system will continue to operate as designed. Generally, the results are only as good as the data used. Ideally, a large set of pass/fail tests or observations to estimate the probability of failure of the item under test would produce the best data. However, this is a costly endeavor if used for every analysis and design.

Developing specific data is costly and time consuming. Instead, analysts rely on available data to assess reliability. Finding data relevant to the specific use and environment for any project is difficult, if not impossible. Instead, we attempt to develop the "best" or composite analog data to support our assessments.

One method used incorporates processes for reviewing existing data sources and identifying the available information based on similar equipment, then using that generic data to derive an analog composite. Dissimilarities in equipment descriptions, environment of intended use, quality and even failure modes impact the "best" data incorporated in an analog composite. Once developed, this composite analog data provides a "better" representation of the reliability of the equipment or component can be used to support early risk or reliability trade studies, or analytical models to establish the predicted reliability data points.

Data that is more representative of reality and more project specific would provide more accurate analysis, and hopefully a better final decision. 